Ágora Rev. Cient. 2018; 05(02):e1

\title{
Percepción de los egresados sobre el proceso enseñanza- aprendizaje en una escuela de enfermería
}

\section{Perception of the graduates on the process teaching learning in a nursing school}

Anika Remuzgo Artezano ${ }^{1}$, Hallder Mori Ramírez ${ }^{2}$

RESUMEN

Objetivo: Determinar e interpretar la percepción de los egresados sobre el proceso de enseñanza-aprendizaje en una Escuela de Enfermería Privada. Materiales y Métodos: El enfoque de la investigación es mixto. El diseño es concurrente según el tiempo de los métodos y Cuantitativo/cualitativo según la prioridad. La muestra estuvo conformada por 120 egresados en la fase cuantitativa, de los cuales 8 fueron seleccionados para la fase cualitativa. El instrumento utilizado en la fase cuantitativa fue una escala de Likert de 30 ítems, validada por juicio de expertos y prueba piloto. Para la fase cualitativa se utilizó una entrevista semiestructurada, la cual permitió agrupar las respuestas en categorías. Resultados: El 57\% de egresados percibe como bueno el proceso de enseñanza - aprendizaje, seguido de un $26 \%$ como regular. En cuanto a las 4 dimensiones, la mayoría percibe como bueno. En la dimensión plan curricular los egresados expresaron disconformidad respecto al plan curricular, expresado que existe inadecuada secuencia de los cursos, respecto a la dimensión estrategias de enseñanza y aprendizaje, la metodología de las sesiones fue con predominio tradicional haciendo uso solo de multimedia. En la dimensión desarrollo de actividades de enseñanzaaprendizaje manifiestan disconformidad con la carencia de oportunidades de prácticas y respecto a la dimensión evaluación del aprendizaje y acciones de mejora expresaron estar de acuerdo con evaluaciones integrales continuas. Conclusión: La mayoría de egresados percibe como bueno el proceso de enseñanza aprendizaje, sin embargo, en el expresan disconformidad en algunas categorías asociadas a las dimensiones.

Palabras clave: percepción, aprendizaje, currículo (DeCS).

\begin{abstract}
Objective: To determine and interpret the perception of the graduates about the teaching-learning process in a School of Private Nursing. Materials and methods: The research approach is mixed. The design is concurrent according to the time of the methods and quant / which according to the priority. The sample consisted of 120 graduates in the quantitative phase, of which 8 were selected for the qualitative phase. The instrument used in the quantitative phase was a Likert scale of 30 items, validated by expert judgment. For the qualitative phase, a semi-structured interview was used, which allowed grouping the responses into categories. Results: $57 \%$ of graduates perceive the teaching - learning process as good, followed by $26 \%$ as regular. As for the 4 dimensions, most perceive it as good. In the curricular plan dimension the graduates expressed disagreement regarding the curricular plan, expressed that there is inadequate sequence of the courses, regarding the teaching and learning strategies dimension, the methodology of the sessions was with traditional predominance making use only of multimedia. In the development dimension of teaching-learning activities, they show disagreement with the lack of internship opportunities and with respect to the evaluation dimension of learning and improvement actions expressed to be in agreement with continuous integral evaluations. Conclusions: the majority of graduates perceive the teaching-learning process as good, however, they express dissatisfaction in some categories associated with the dimensions.
\end{abstract}

Key words: perception, learning, curriculum (DeCS).

${ }^{1}$ Coordinadora Académica. EAP de Enfermería. Universidad Privada Norbert Wiener. Lima - Perú

${ }^{2}$ Profesor Principal TC. Facultad de Medicina. Universidad Nacional Mayor de San Marcos. Lima - Perú. 


\section{INTRODUCCIÓN}

La educación constituye un factor clave en el desarrollo de los países, generando un capital humano capaza de contribuir al alcance de un mayor crecimiento económico y desarrollo sociocultural (1).

La educación y la universidad en el Perú, por un largo periodo, sentaron sus bases en un modelo pedagógico tradicional. Producto de ello, surgieron los patrones de aprendizaje pasivos, receptivos y memorísticos, siendo el conocimiento transmitido asumido como una verdad absoluta. Así mismo, se generó un esquema de enseñanza instructivista, donde el docente monopoliza el conocimiento y juega un rol activo en la transmisión del mismo. El alumno juega un rol secundario, teniendo una función meramente receptiva de conocimiento (2).

El devenir de las universidades en los últimos años, han dado origen a una gran heterogeneidad y masificación, creando sistemas de enseñanzaaprendizaje tan dispersos que derivan en una baja calidad educativa. Por ello, una de las preocupaciones centrales de las universidades en el Perú, es la calidad educativa, así como los sistemas que permitan una evaluación y acreditación institucional (3).

Son muchos los factores asociados a esa preocupación, la amplia cobertura, masificación de instituciones y los diversos modos de producir conocimiento (4).

La calidad educativa universitaria, es definido a través de diversos enfoques, interpretaciones y dimensiones, por ello resulta complejo tener un concepto unificado. Sin embargo, un aspecto relevante es vincular a la calidad educativa con la pertinencia y el impacto, resultando difícil concebir universidades de calidad sin una pertinencia para su entorno social (5).

La carrera profesional de Enfermería, no ha sido ajena a dicha problemática, puesto que requiere una revisión constante, para que se encuentre acorde a los cambios teóricos y prácticos actuales que generen profesionales con una visión holística y realista del entorno en que se desenvuelven. Para ello, se requieren cambios en la estructura de los programas de formación y mallas curriculares que orienten a resolver problemas de la realidad (6).

Por lo anterior, La educación superior en el Perú, incluido la carrera profesional de enfermería, inició hace algunos años un proceso de revisión y análisis a profundidad de la realidad universitaria nacional.

En ese sentido el Perú, a través del Consejo Superior del Sistema Nacional de Evaluación,
Acreditación y Certificación de la Calidad Educativa (SINEACE) y su órgano operador el Consejo de Evaluación, Acreditación de la Educación Superior (CONEAU) constituye importantes organismos encargados de garantizar la calidad educativa (7).

En el año 2014, se promulgó la ley 30220, declarándose la reorganización del SINEACE. En el marco de dicha reorganización se recomendó un nuevo modelo de acreditación, que conciba la evaluación de la calidad como un proceso formativo brindando oportunidades de mejora progresivas y sostenidas en el tiempo, a las instituciones universitarias. Dicho modelo presenta 4 dimensiones: gestión estratégica, formación integral, soporte institucional $\mathrm{y}$ resultados(8).

En ese contexto, hasta la fecha se ha logrado acreditar en diversas universidades públicas y privadas, la carrera profesional de enfermería, jugando un rol importante la percepción de los estudiantes sobre el proceso de enseñanzaaprendizaje (9). La Escuela Académico Profesional de Enfermería de la universidad privada donde se desarrolla la investigación, fue acreditada según los estándares de SINEACE el 2015.

En la presente investigación, se ha tomado como modelo, el elaborado por el Consejo Nacional de Evaluación y Acreditación Universitaria (CONEAU), en el cual mencionan a la dimensión formación profesional, que tiene como factor al proceso de enseñanza aprendizaje y cuyos criterios de evaluación son: el plan curricular, estrategias enseñanza-aprendizaje, desarrollo de actividades y evaluación del aprendizaje (10).

Desde una perspectiva empírica se observa que los estudiantes se preguntan si el plan curricular es el idóneo para su formación e incluso se oye comúnmente cuando se les pregunta sobre lo aprendido en los 8 ciclos, previos al internado, y refieren expresiones de asombro tales como: "¿Por qué pasé de ciclo?, "no me siento preparado", "debieron ser más estrictos, cuando me evaluaban", "no debí haber aprobado", "aún nos falta mucho".

Al respecto, Palomino Taquire R., en el año 2018, en Cusco-Perú, realizó la tesis titulada "Percepción de las actividades de enseñanza aprendizaje en los estudiantes durante la especialización en enfermería en una universidad privada de Cusco", utilizó para ello un diseño descriptivo-transversal, encontrando en su mayoría de estudiantes una percepción medianamente favorable (11). 
Quispe Ramos L., en el año 2018, en Lima Perú, realizó la tesis titulada "Experiencias en la enseñanza - aprendizaje del proceso de atención de enfermería en estudiantes de noveno ciclo de una universidad privada - Chiclayo, 2017" utilizando un diseño cualitativo de investigación, tipo abordaje de estudio de caso. La muestra estuvo conformada por 16 estudiantes y 14 docentes, el instrumento utilizado fue una entrevista semiestructurada. Los resultados obtenidos fueron: no existe uniformidad en la enseñanza del proceso de atención de enfermería (PAE), dificultades en la aplicación en la práctica, necesidad urgente de estandarizar la enseñanza de PAE (12).

Pecina R, en el año 2015, en México, realizó la investigación titulada "Percepción del estudiante de enfermería sobre la calidad del proceso enseñanza - aprendizaje" utilizando un diseño de investigación cualitativo. La muestra estuvo conformada por 28 estudiantes de enfermería, el instrumento utilizado fue una entrevista semiestructurada. Las conclusiones fueron que existe diversas percepciones referidas al proceso de enseñanza - aprendizaje, las cuales se agrupan en la mejora de los procesos académicos y una adquisición parcial de competencias establecidas en los perfiles del egresado (13).

En la revisión bibliográfica, se evidencia que la mayoría de estudios utilizan un enfoque cuantitativo o cualitativo, siendo escasos los que utilizan un enfoque mixto de la investigación. Al ser de naturaleza compleja el problema de investigación, y estar conformada por una realidad objetiva y subjetiva, es conveniente capturar ambas percepciones de los estudiantes de enfermería, motivo por el cual se utiliza un enfoque mixto, siendo un importante aporte metodológico.

En este contexto, se plantean las siguientes interrogantes: ¿Será que el Plan Curricular desarrollado, no es adecuado y necesita ser modificado?, ¿Cuáles son las Características de las estrategias de enseñanza aprendizajes? Dichos cuestionamientos se hacen necesarios responder con la finalidad de mejorar la calidad educativa en la carrera Profesional de Enfermería, para que dichos profesionales puedan brindar cuidados a la población y logren así el fin supremo de una universidad, aportar soluciones a los problemas de salud de la sociedad.

La investigación tiene por objetivo determinar e interpretar la percepción de los egresados de una Escuela de Enfermería sobre el proceso enseñanza aprendizaje. Los objetivos específicos buscan determinar la percepción de las 4 dimensiones del proceso de enseñanza-aprendizaje: plan curricular, estrategias de enseñanza aprendizaje, desarrollo de actividades.

La importancia de la investigación se basa en los resultados obtenidos, los cuales sirven de insumo importante para que la institución universitaria pueda realizar un análisis crítico del proceso de enseñanza-aprendizaje $\mathrm{y}$ tomar decisiones al respecto.

\section{MATERIALES Y MÉTODOS}

El enfoque de estudio de la investigación es mixto. El enfoque mixto integra los enfoques cuantitativos y cualitativo. Al respecto, existen diversos autores que han planteado las justificaciones para su uso, siendo las principales: verificar o contrastar los datos cuantitativos y cualitativos, obtener una comprensión más integral del problema, tener una mayor capacidad de explicar el problema, refuerza la credibilidad de los resultados (14). Dicho enfoque se eligió por la complejidad del problema de investigación. En cuanto al diseño de investigación, se utilizó un diseño concurrente, puesto que fueron aplicados los métodos cuantitativos y cualitativos de manera simultánea (15). Según prioridad, es CUAN/cual, debido a que la muestra mayor es cuantitativa (14).

La población del estudio estuvo conformada por 120 egresados de la Escuela Académico Profesional de Enfermería de una universidad privada para la fase cuantitativa, de los cuales de conformó un grupo de 8 egresados seleccionados aleatoriamente para la fase cualitativa. Para la fase cuantitativa, se trabajó con toda la población de egresados, y para la fase cualitativa se realizó un muestreo anidado, porque representa una porción de la muestra cuantitativa (16).

Los criterios de inclusión fueron: egresados que hayan culminado el X semestre académico y acepten en forma voluntaria, participar en el estudio y a la entrevista a profundidad. Los criterios de exclusión fueron: egresados, que no asistan el día de la aplicación del instrumento y no acepten participar en las entrevistas a profundidad a través de los grupos focales.

La técnica utilizada para la fase cuantitativa fue la encuesta y la para la fase cualitativa fue la entrevista semiestructurada a un grupo focal.

El instrumento utilizado para la fase cuantitativa fue una escala de Likert, que consta de 30 ítems, los cuales representan las 4 dimensiones de la variable principal. El tiempo de aplicación del instrumento es de 10 a 15 minutos. Respecto a la validez, fue sometido a juicio de expertos, que resultó con un nivel de concordancia 
significativo $(p=0.0212)$. La confiabilidad fue evaluada mediante una prueba piloto, en una muestra con características similares al estudio, resultando un Alpha de Crombach de 0.9.

El instrumento para la fase cualitativa fue una ficha de entrevista semiestructurada, que permitió profundizar en el estudio de las dimensiones de la variable.

Una vez recolectados los datos cuantitativos, se creó una matriz de los mismos en el programa Microsoft Excel 2013, además se asignaron puntuaciones para cada una de las respuestas y fueron clasificados mediante categorías.

Para los datos cualitativos, en concordancia con el cronograma presentado se realizó la recolección de datos, las entrevistas se desarrollaron en un promedio de 1 hora a 8 egresadas seleccionadas en forma aleatoria y voluntaria por el gran aporte que representaban cada una de ellas. Un primer contacto se llevó a cabo para solicitar su colaboración explicando los objetivos y de esa manera conocer su aceptación, y definir la fecha para la entrevista y el lugar respectivo.

En el análisis e interpretación de datos se realizaron las transcripciones de los datos, luego de una o varias lecturas se establecieron las unidades de análisis de los datos, asignándoles códigos de análisis, posteriormente se reunieron los códigos similares para estructurar las categorías y sub-categorías. Estas categorías fueron interpretadas a la luz de las teorías o autores seleccionados por el investigador, que tratan sobre el tema en estudio. En esta fase del estudio emergieron cuatro categorías, las cuales fueron interpretadas en base al modelo seleccionado por el autor, en el planteamiento del problema.

Durante el proceso de la investigación, los 4 principios bioéticos de la investigación (autonomía, beneficencia, no maleficencia y justicia) (17) sirvieron de guía para garantizar la confidencialidad, el consentimiento informado y evitar daños a los participantes.

\section{RESULTADOS}

Los resultados sociodemográficos mostraron las características de los egresados de enfermería de una universidad privada que participaron en el estudio, todos se residían en Lima, Perú. Las mujeres representaron el $85 \%(n=102)$ de la población. Las edades estuvieron comprendidas entre los 20 a 50 años con una edad promedio de 29,7 y desviación estándar de 5,2 años; según grupo de edad de 30 a 34 tuvieron el mayor porcentaje de representación con $40 \%$, seguido del grupo de 25 a 29 años con $32,5 \%$, el grupo de 20 a 24 años con $12,2 \%$ y mayores de 35 años $13,3 \%$.

Respecto a los resultados cuantitativos, se encontró que: la percepción general, del proceso de enseñanza aprendizaje desarrollado en su formación académica (Grafico 1), el 15\% tiene una excelente percepción, el $57 \%$ una buena percepción, $26 \%$ regular y $2 \%$ mala percepción.

En la dimensión plan curricular, se obtuvo un nivel de percepción excelente en el $25 \%$, bueno en el $48.3 \%$, regular en el $22.5 \%$ y malo en el $4.2 \%$. En la dimensión estrategias de enseñanzaaprendizaje, se obtuvo un nivel de percepción excelente en un $17.5 \%$, bueno el $42.5 \%$, regular en el $34.2 \%$ y malo en el 5.8\%. En la dimensión desarrollo de actividades de aprendizaje se obtuvo un $48.3 \%$ percepción buena, $40.8 \%$ regular, $10 \%$ excelente y $8 \%$ malo. En la dimensión evaluación se obtuvo un $45.8 \%$ percepción buena, $29.2 \%$ excelente, $24.2 \%$ regular y $8 \%$ malo.

Los resultados reflejan que mayoritariamente los alumnos egresados de la escuela de enfermería de una universidad privada, perciben al proceso de enseñanza-aprendizaje y sus dimensiones como bueno. Dichos resultados deben ser contrastados con la fase cualitativa.

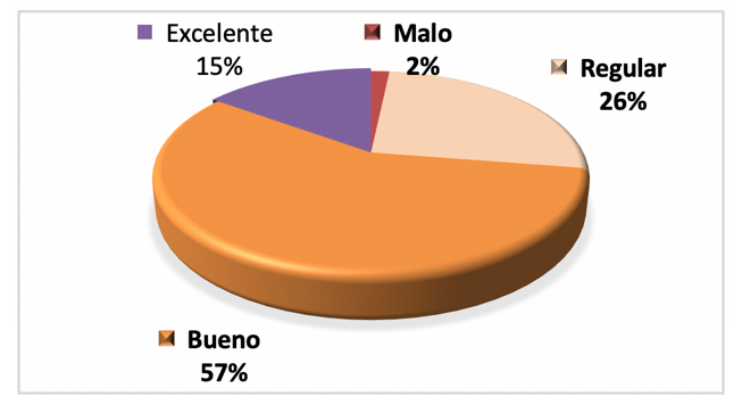

Grafico 1: Percepción del proceso enseñanza aprendizaje de los egresados de enfermería en una universidad Privada de Lima. $(n=120)$.

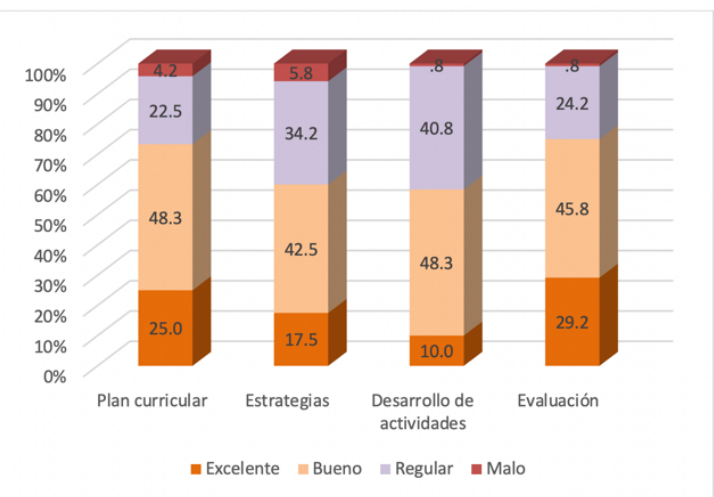

Grafico 2: La percepción del proceso enseñanza aprendizaje según dimensiones de los egresados de Enfermería en una universidad Privada de Lima 2018. 
Los resultados cualitativos fueron caracterizados mediante los 4 indicadores estructurados en el factor de enseñanza - aprendizaje, fundamentados en el Marco conceptual del Modelo de CONEAU. Se presentan los principales resultados:

Dimensión Plan Curricular: Los egresados han manifestado una posición intermedia, debido a las variaciones que ha presentado el Plan curricular, lo cual han considerado positivo para su desarrollo profesional, refieren

“...bueno mira que ha ido avanzando los cursos de anatomía, filosofia, que ya no hemos podido vivir la nueva currícula que hicieron de morfosis, tampoco he podido, como es que asi lo llevan, pero en cuanto a lo que nosotros, yo que he llevado mi curso por separado de anatomía $y$ fisiología, también he podido aprender mucho, utilizábamos los laboratorios.... (P-03)”

Asimismo, consideraron necesario que dentro de la currícula debe haber cursos de especialización, que permita el desarrollo de procedimientos en el ámbito clínico.

“......para mi es primordial, eso, el otro punto que estaba viendo es, lo que mencionaba mi compañero, no, que no hay cursos de especialización, si bien es cierto la currícula solamente nos enseñan a nosotros lo básico en enfermería, pero creo yo que, también debería por ahi implementarse, este, cursos de especialización, pero que estos también sean básicos para nosotros también centrar a tallar ahí en nuestras prácticas hospitalarias, de clínicas, en el internado, que se yo, no, para poder tener conocimiento, y no decir, no, eso no nos enseñan, .....(P-06)."

Dimensión Estrategias de enseñanza-aprendizaje: en los resultados cuantitativos se obtuvo que la percepción que tienen los egresados en relación a la dimensión Estrategias de Enseñanza con tendencia a regular.

Técnicas de aprendizaje

“... tenemos los equipos multimedia, mas recreativo, el alumno no se siente tan aburrido, o es dinámico, mas didáctico...(p-02)",

En relación al ítem 16 el 87\% de egresados está totalmente de acuerdo. En relación a ello refieren. “....ahora ya se hace más esquemas, sketch, se hace dramatización, y eso es bueno, porque el alumno ya aprende más, no solamente con miras las ayudas audiovisuales y el pizarrón, sino también que dramatiza lo que se va hacer,.. (P06)"

Dinamismo vs no dinamismo

En relación al ítem 18 el 82\% de egresados está de acuerdo a totalmente de acuerdo. En relación a ello refieren.

“... ya luego de ello, en los cursos de carreras, la mayoría de profesores si eran, este, didácticos, muy didácticos, hacían ejercicios, salías adelante, pero también habian algunos profesores que más que nada era por, por la misma teoría que era, digamos las características del curso, ,.. (P-03)”

Dimensión desarrollo de las actividades de enseñanza-aprendizaje: En relación al ítem N²4 Algunas sedes de práctica carecían de oportunidades y buena infraestructura para mi aprendizaje". el 59\% estuvo en desacuerdo y el 41 $\%$ de acuerdo de los egresados. En relación a los que están de acuerdo refieren.

Estrategias generadoras de oportunidades de aprendizaje

“...eso escucho que lo conceptualizan a la interna, ella, llegabas ni bien llegabas, toma el estetoscopio, toma el termómetro, contrólame las funciones vitales a todos los pacientes, entonces creo que, de alguna u otra forma, tratar de los que vienen, se puede hacer de que, poder darle más campo, más oportunidades en el sitio, , ,.. (P-03)”

Dimensión Evaluación del aprendizaje y acciones de mejora: En relación al ítem $\mathrm{N}^{\circ} 29$, el sistema de evaluación que aplico el docente en el aula fue continuo a través de talleres, trabajos grupales, argumentos, etc., que permitió identificar mis logros y superar mis dificultades en forma oportuna. el $8 \%$ está en desacuerdo y el $92 \%$ de acuerdo de los egresados.

En relación a los que están desacuerdo refieren.

“...ahora te piden producto, ahora te piden examen, ahora te piden asistencia, si no asistes $30 \%$, eh, te suspendian por asi decir de las clases, antes no era así, antes la forma de evaluar era distinto, .. (P-01)" 


\section{CONCLUSIONES}

En relación a los objetivos cuantitativos, la mayoría de egresados tiene una buena percepción del proceso enseñanza-aprendizaje y sus dimensiones. En cuanto a los objetivos cualitativos, los egresados perciben respecto al proceso de enseñanza aprendizaje: Disconformidad respecto al plan curricular, expresado cuando refieren que existe inadecuada secuencia de los cursos y ausencia de algunos considerados importantes como los cursos de carrera. Respecto a las estrategias de enseñanza y aprendizaje, la metodología de las sesiones fue con predominio tradicional haciendo uso solo de multimedia. Respecto al desarrollo de las actividades de enseñanza aprendizaje refieren disconformidad en la metodología de la enseñanza llamándola expositiva. Evaluación del aprendizaje y acciones de mejora, refieren poca objetividad del proceso de evaluación del aprendizaje. Finalmente, los egresados de enfermería son fuente de información muy importante para evaluar los procesos de enseñanza-aprendizaje, dicha información es resultado de los años de aprendizaje y las percepciones de sus experiencias durante su paso por las aulas universitarias.

\section{AUTOR DE CORRESPONDENCIA}

Mg. Anika Remuzgo Artezano

Escuela de Enfermería

Universidad Privada Norbert Wiener

Av. Arequipa 444. Cercado de Lima 15046

Teléfono: 991266187

E-mail: anika.remuzgo@uwiener.edu.pe

\section{REFERENCIAS BIBLIOGRÁFICAS}

1. Ministerio de Educación del Perú. La Universidad en el Perú: Razones para una reforma universitaria Informe 2006 [Internet]. Lima- Perú; 2006. 1-198 p.

2. Mejía J. El proceso de la educación superior en el Perú. La descolonialidad del saber universitario. Rev Investigaciones Sociales [revista en Internet] 2017 [acceso 23 de setiembre de 2018]; 21(38): 199-212.

3. Cevallos D. La Calidad Educativa en la realidad Universitaria Peruana frente al Contexto Latinoamericano. Rev Flumen [revista en Internet] 2014 [acceso 8 de setiembre de 2018]; 7(1): 3-8.

4. Tello C, Medina J, Ishikane G. Evaluación de la Carrera Profesional de Enfermería:
Percepción de los estudiantes en una Universidad Nacional del Perú . Rev Enferm Herediana [revista en Internet] 2017 [acceso 8 de abril de 2018]; 10(1): 63-70.

5. Baca A, León M, Mayta J, Bancayán O. Aseguramiento d la calidad de la formación universitaria en el Perú. Rev Interuniversitaria de Formación del Profesorado [revista en Internet] 2014 [acceso 8 de junio de 2018]; 49(17): 35-48.

6. Vallejo N. Desarrollo de la Enfermería. Rev Peru Med Exp Salud Publica [revista en Internet] 2002 [acceso 8 de abril de 2018]; 36(21): 347-367.

7. Ministerio de Educación del Perú. Reglamento de la Ley No 28740 , Ley del Sistema Nacional de Evaluación , Acreditación y Certificación de la Calidad Educativa [Internet]. Lima - Perú: Ministerio de Educación del Perú; 2007. 1-23 p.

8. SINEACE. Modelo de Acreditación para Programas de Estudios de Educación Superior Universitaria Octubre 2016 [Internet]. Lima- Perú; 2016. p. 36.

9. Yamada G, Castro J. Calidad y acreditación de la educación superior: retos urgentes para el Perú [Internet]. Lima-Perú: Universidad del Pacífico; 2013. 216 p.

10. Ministerio de Educación del Perú. Guía para la acreditación de carreras profesionales universitarias del CONEAU 2012 [Internet]. Lima- Perú; 1-47 p.

11. Palomino R. Percepción de las actividades de enseñanza - aprendizaje en los estudiantes durante la especialización en enfermería en una universidad privada de Cusco [tesis maestría]. Perú: Universidad Peruana Cayetano Heredia; 2018 [Internet].

12. Quispe L. Experiencias en la enseñanza aprendizaje del proceso de atención de enfermería en estudiantes de noveno ciclo de una universidad privada - Chiclayo, 2017 [tesis Licenciatura]. Perú: Universidad Católica Santo Toribio de Mogrovejo; 2018 [Internet].

13. Pecina R. Percepción del estudiante de enfermería sobre la calidad del proceso de enseñanza aprendizaje. Rev Investigaciones, estrategias y medios en la práctica educativa [revista en Internet] 2015 [acceso 8 de febrero de 2018]; 22(2): 222-229.

14. Hernández R, Fernández $C$, Baptista $P$. Metodología de la Investigación. 6a ed. México D. F.: McGraw-Hill; 2014.

15. Guelmes E, Nieto L. Algunas reflexiones sobre el enfoque mixto de la investigación pedagógica en el contexto cubano. Rev 
Universidad y Sociedad [revista en Internet] 2015 [acceso 8 de julio de 2018]; 7(2): 2329.

16. Moscoso JN. Los métodos mixtos en la investigación en educación: hacia un uso reflexivo. Cadernos de Pesquisa [revista en Internet] 2017 [acceso 8 de febrero de 2018]; 47(164): 632-649.

17. Arellano J, Hall R, Hernández J. Ética de la Investigación Científica. México:Universidad Autónoma de Querétaro; 2014. [Internet].

18. Serrano-Gallardo $P$, Martínez-Marcos $M$, Espejo-Matorrales F, Arakawa T, Magnabosco GT, Pinto IC. Factores asociados al aprendizaje clínico de los estudiantes de enfermería en la atención primaria de salud: un estudio transversal analítico.Revista Latino-Americana de Enfermagem [revista en Internet] 2016 [acceso 8 de febrero de 2018]; 24(2): 1-10.

19. Gómez R, Díaz B, Fernández I, Naithe D. Percepción de estudiantes sobre el proceso enseñanza aprendizaje en la asignatura de Enfermería Pediátrica. Revista Habanera de Ciencias Médicas [revista en Internet] 2016 [acceso 8 de febrero de 2018]; 15(4): 630641.

20. Jiménez M, Casas L, Luengo R. Representación del conocimiento y percepción subjetiva del proceso de aprendizaje profesional: estudio cualitativo en personal de enfermería. Rev Educ Med [revista en Internet] 2010 [acceso 8 de febrero de 2018]; 13(3): 163-170.

21. Ledesma M. Análisis de la Teoría de VYGOTSKY para la Reconstrucción de la Inteligencia Social I [Internet]. Cuenca Ecuador: Universidad de Cuenca; 2014. 126 p. 